\title{
Olimpiada de lengua para sordos una experiencia lingüística y educativa
}

Olympic for deaf peaople, a linguistic and educational experience

Recibido el 2/ 09/2019. Revisión del 7/09 al 25/ 10/2019. Aceptado 27/10/2019

Ludmilan Zambrano Steensma

lzambrano@usdg.edu.pe https://orcid.org/0000-0003-4347-0398

Universidad Santo Domingo de Guzmán de Perú

Luis Alejando Díaz ldiaz66ve@gmail.com

iD https://orcid.org/0000-0002-2874-6213 Universidad Nacional Experimental de la Seguridad

\section{Resumen}

En Venezuela, las personas sordas cuentan con el reconocimiento de la lengua de señas venezolana (LSV) que los constituye como comunidad lingüística minoritaria. Las investigaciones acerca de las producciones en LSV y la relación de las personas sordas con el español oral y escrito, se han incrementado, de donde surgió la investigación que se reporta en este artículo y cuyo objeto fue estudiar la situación de las unidades educativas del área de Educación Especial y la discapacidad auditiva, en relación con el español escrito, para organizar actividades que estimulen su uso como segunda lengua en los niños y jóvenes sordos. Para ello el método fue la investigación acción participante, se partió de un diagnóstico de la situación, que dio cuenta de las dificultades en la relación espontánea de los Sordos con el código escrito, por tanto, se planificó la Olimpiada de lengua para niños y jóvenes sordos en el estado Aragua, se ejecutó y evaluó su impacto en la comunidad. La Olimpiada tuvo como objetivo fundamental, estimular el uso de los textos escritos en español por niños y jóvenes sordos; propiciar su participación en actividades de producción y comprensión de textos escritos y en LSV a partir de distintos contextos lingüísticos. Al evaluar la ejecución de la Olimpiada se obtuvo como resultado la participación activa de los niños y jóvenes sordos en la producción de textos a partir de la narración y lectura de cuentos; la comprensión de narraciones, expresadas en forma escrita, lengua de señas y la dramatización.

Palabras clave: segunda lengua, español, innovación, sordos 


\begin{abstract}
In Venezuela, deaf people have the recognition of the Venezuelan Sign Language (LSV) that constitutes them as a minority linguistic community. Research on productions in LSV and the relationship of deaf people with oral and written Spanish, have increased, where the research that is reported in this article and whose purpose was to study the situation of educational units in the area Special Education and hearing impairment, in relation to written Spanish, to organize activities that encourage its use as a second language in children and young deaf people. For this, the method was the participant action investigation, it was based on a diagnosis of the situation, which gave account of the difficulties in the spontaneous relationship of the Deaf with the written code, therefore, the Language Olympiad for children and young people was planned deaf in the state of Aragua, its impact on the community was executed and evaluated. The Olympiad had as its main objective, to stimulate the use of texts written in Spanish by deaf children and young people; promote their participation in production activities and comprehension of written texts and LSV from different linguistic contexts. When evaluating the execution of the Olympiad, the active participation of deaf children and young people in the production of texts based on storytelling and storytelling was obtained; the understanding of narratives, expressed in written form, sign language and dramatization.
\end{abstract}

Keywords: second language, Spanish, innovation, deaf

\title{
Introducción
}

La historia de la educación especial en Venezuela ubica a las personas sordas en una dimensión de respeto a sus necesidades comunicativas y condición lingüística como comunidad, a partir de la visión socioantropológica contenida en el Modelo de Atención Educativa Integral del Deficiente Auditivo Venezolano y puesta en marcha por el Ministerio de Educación en 1998 (ME, 1998a). No obstante, la aplicación del modelo no satisface completamente las necesidades e intereses de los estudiantes sordos en términos de su relación con los textos escritos en español, debido a los factores sociolingüísticos, educativos y políticos que han influido negativamente en el fortalecimiento de una educación verdaderamente bilingüe-bicultural, lo cual ha generado nuevas propuestas y alternativas de atención para las personas sordas que promuevan cambios en la visión y misión de la educación especial y que todavía no se han oficializado. 
De allí que esta investigación se propuso la indagación del contexto educativo en el estado Aragua en el área de la sordera, en relación con el uso del español escrito como segunda lengua en el marco del proceso de alfabetización de las personas sordas en las escuelas del área. Por ello, con base en el diagnóstico realizado para tal fin, se llevó a cabo la planificación y ejecución de la Primera Olimpiada de Lengua para niños y jóvenes Sordos en el estado Aragua (entre Octubre 2012 y Julio 2013) con el objeto de propiciar la producción y comprensión de textos escritos en español con base en el respeto a la lengua de señas a través de un contexto de aprendizaje significativo y estimulante.

Estas Olimpiadas se llevaron a cabo con la participación de la población estudiantil de las unidades educativas correspondientes al área de Sordos en el estado Aragua, ubicadas en el Municipio Libertador y Girardot. Para ello se contó con el personal docente oyente y sordo, padres, representantes y comunidad en general, con quienes a través de la formación e información se organizaron las jornadas de trabajo para acercar los textos escritos en español a la comunidad escolar con discapacidad auditiva.

Las actividades se justifican ampliamente en el aspecto sociolingüístico, por cuanto contribuyeron con el uso de la lengua de señas y el español escrito en los niños y jóvenes participantes; en el aspecto socioafectivo, propiciaron el acercamiento de los niños y jóvenes con sus padres, representantes, docentes y personal en general, quienes no solo apoyaron la preparación de los niños para su participación en las actividades, sino que también se involucraron en el logro de los objetivos; desde el punto de vista pedagógico, se presentan actividades innovadoras en el aula, que propician la creación de nuevas actividades y propuestas de los docentes, quienes colaboraron con entusiasmo en logro de los objetivos.

Así mismo, esta investigación se concibió como una oportunidad para el fomento de actividades de investigación, formación, información y documentación en el campo de la educación para las personas sordas (de acuerdo con la Ley para personas con discapacidad, 2007) y como una contribución para facilitar el uso del código escrito en actividades de promoción y difusión de la lectura y la escritura significativa en niños y jóvenes sordos a través de la lengua de señas. La investigación procuró el respeto a la igualdad, la justicia y la solidaridad como valores que orientan el trabajo educativo. 
En atención al derecho que asiste a los niños, jóvenes y adolescentes sordos, se realizó el diagnóstico, planificación, ejecución y evaluación de la Primera Olimpiada con la participación de 60 niños y jóvenes pertenecientes a la Primera Etapa de Educación Primaria para sordos; 30 docentes y 8 estudiantes universitarios de educación especial en el área de la sordera, además de los docentes y auxiliares de las dos dependencias educativas y el personal directivo de cada una de ellas.

Los estudiantes universitarios y la investigadora llevaron a cabo el abordaje pedagógico en las escuelas junto a los docentes de aula. Luego de la participación activa de las comunidades educativas de ambas instituciones, se presenta el resultado de este proceso bajo la metodología de investigación acción participante, que involucró la colaboración de todos los actores significativos e interesados en el hecho educativo. Se desarrolló bajo esta premisa metodológica a partir de un plan de acción, con el fin de facilitar la igualdad de oportunidades y condiciones para los niños y jóvenes sordos, sus padres y sus docentes.

De acuerdo al diagnóstico elaborado como parte del estudio, el aspecto más relevante fue la preocupación de los docentes, padres y representantes e inclusive auxiliares de las instituciones educativas, ante la poca o escasa relación de los niños y jóvenes Sordos con la lengua escrita (Sánchez, 2014) y la ausencia de actividades innovadoras que estimulen su uso en contextos escolares y sociales. Esta situación generó la propuesta de un plan de acción dirigido a organizar una acción innovadora para sordos. El objetivo general del plan fue proponer la I Olimpiada de lengua como parte de un plan de acción que involucre actividades que estimulen el uso de la lengua escrita en los niños y jóvenes del área de sordos, a partir de la información recabada.

\section{Materiales y métodos}

En la construcción del diagnóstico e identificación de la problemática, se llevaron a cabo las entrevistas a los miembros del personal directivo; el registro de las conversaciones (Teppa, 2006; Hurtado y Toro, 1997), sostenidas en diversas oportunidades con el personal docente; así como la revisión y análisis de documentos e investigaciones relacionadas con la educación de niños, niñas y adolescentes sordos. 
Para recabar la información se diseñó un guión de entrevista semiestructurada y un registro de los principales aspectos discutidos y abordados en las conversaciones sostenidas en las insituciones con los diferentes actores involucrados en el quehacer educativo. De allí se desprendió la matriz FODA (Fortalezas, oportunidades, debilidades y amenazas). Cada una de las instituciones presenta características propias, disímiles en algunos aspectos y coincidentes en otros. Cabe destacar en ambos casos la mística y dedicación del personal directivo, docente, auxiliar, administrativo y obrero, quienes, con su empeño y compromiso, contribuyen día a día con la formación y educación integral que reciben los niños, niñas y jóvenes en estas unidades educativas.

Aunque ambas instituciones tienen realidades geográficas y de infraestructura disímiles, el punto focal de este trabajo, no radica allí, en el aspecto físico mueble e inmueble, sino en el aspecto académico y pedagógico. En la indagación de ambos contextos de estudio, coincide la forma de abordar el uso del código escrito como segunda lengua en los niños y jóvenes sordos, la ausencia de actividades que estimulen el uso del código escrito, aunado al escaso conocimiento y comunicación mediante la lengua de señas venezolana. Esta situación guarda relación con las observaciones de aula realizadas y otros trabajos de investigación (Luque, 1994; Morales y Vallés, 2002) que dan cuenta de la escasa o difícil vinculación que establecen las personas sordas con el español escrito como segunda lengua en el ámbito educativo y en la vida cotidiana.

De acuerdo con la información suministrada por los miembros de ambas instituciones educativas, aún no se reporta la consolidación del español escrito como segunda lengua a lo largo de la historia educativa de los sordos venezolanos, a pesar de los esfuerzos y las investigaciones que dan cuenta de la relación que guardan las personas sordas con el español escrito y el cambio paradigmático y operativo que implicó la transformación constante de la praxis educativa.

Por ello, las ciencias sociales requieren de enfoques novedosos, dinámicos, que den cuenta de la realidad educativa y en las interacciones producto del complejo espacio donde se forman las futuras generaciones: las escuelas. De allí que el método de investigación acción, involucra una nueva visión del hombre y de la ciencia (Martínez, 2011), ya que se resiste contra el Ethos positivista, pues implica un proceso de emancipación y desarrollo de los seres humanos.

Esta forma de investigar se apoya en la racionalidad hermenéutica, que significa de acuerdo con Cisterna (2005:62), una forma de abordar, estudiar, entender, analizar y construir conocimiento a 
partir de procesos de interpretación, donde la validez y confiabilidad descansa en el rigor del investigador. Mediante el uso del lenguaje como hilo conductor del acto hermenéutico, pues por una parte hace posible la construcción social de la realidad y, por la otra, posibilita el entendimiento humano desde su esencia dialéctica.

El sustento metodológico de la investigación que se desarrolló fue la investigación-acción participante y Martínez (2011) la define como:

La investigación- acción participante realiza simultáneamente la expansión del conocimiento científico y la solución de un problema, mientras aumenta igualmente la competencia de sus respectivos participantes (sujetos coinvestigadores) al mejorar al ser llevada a cabo en colaboración, en una situación concreta y usando la realimentación de la información en un proceso cíclico (p. 97).

En este método de investigación no se siguen pasos previamente establecidos, que se deban continuar con rigidez, ya que como proceso dinámico se puede abordar de diversos modos (Hurtado y Toro, 1997). A los efectos y fines de la investigación y en atención a la experiencia de los investigadores, se tomaron en cuenta los siguientes elementos:

La población total del trabajo de investigación estuvo conformada por los niños y jóvenes sordos, pertenecientes a las unidades educativas de Educación Especial en el área de Educación para sordos del estado Aragua para el año escolar 2012-2013. Esta población estuvo conformada por un total de 386 niños y jóvenes sordos. Para la investigación se contó con la participación de dos instituciones, una ubicada en el Municipio Girardot cuya población escolar fue de 166 y la otra ubicada en el Municipio Libertador fue de 34; para un total poblacional de 190 niños, niñas y jóvenes sordos.

\section{Informantes}

Para la organización de la muestra se consultó con el personal directivo y docente de las instituciones educativas, durante la organización de la Olimpiada, se acordó con las instituciones educativas, trabajar por categorías de edades y no por grados, quedando de la siguiente manera: Grupo "A" de 6 a 8 años; Grupo "B" de 9 a 11 años y Grupo "C" de 12 en adelante. De acuerdo con este criterio el total de niños participantes fue de 39 niños, niñas y jóvenes pertenecientes a ambas instituciones. 
También se debe destacar la participación y colaboración de los ocho (8) estudiantes universitarios; de los padres y representantes de los niños, niñas y jóvenes sordos; la Asociación Polideportiva de Sordos del estado Aragua; las Unidades Educativas Nacionales de Educación Especial, del Área; el personal Intérprete de Lengua de Señas Venezolana de la universidad.

\section{Técnicas e Instrumentos}

Para recabar la información básica para la construcción del diagnóstico que sustentó el plan de acción, la técnica empleada fue la entrevista, para lo cual se diseñó como instrumento un guión de entrevista semiestructurada. Este instrumento se administró tanto al personal directivo como a los docentes de los planteles participantes, así como también se complementó con las conversaciones desarrolladas con el personal auxiliar sordo y oyente, los niños, jóvenes y las observaciones de aula. Se hicieron las notas de campo (Martínez, 1999), para reportar y registrar cada actividad realizada.

Dada la calidad humana y de servicio de todo el personal, de los padres y representantes, de los niños y de los miembros de la comunidad, se pudo recabar la información necesaria, que permitió identificar las situaciones problemáticas, jerarquizarlas y buscar los puntos de encuentro entre las situaciones presentes en cada plantel. De acuerdo con Martínez (2011, p. 240), el investigador actúa esencialmente como un organizador de las discusiones, como un facilitador del proceso, como un catalizador de problemas y conflictos; de allí que contribuyó positivamente en la planificación, desarrollo y concreción de los objetivos propuestos, que los investigadores establecieron el puente de comunicación en cada una de las instituciones, lo cual permitió la empatía y la confianza entre los participantes requisito indispensable para desarrollar la investigación acción participante.

En cuanto al registro del desarrollo del plan de acción se empleó como técnica el video, la observación y las fotografías y; como instrumento, las notas de campo para reportar las actividades desarrolladas durante la puesta en práctica de las acciones organizadas en relación con la Olimpiada de lengua para sordos en el estado Aragua.

\section{Fases de la Investigación}


Con base en los conceptos y aportes de Martínez (1999, 2011); Hurtado y Toro (1997), se establecieron como momentos, fases o etapas de la investigación los pasos que se describen seguidamente:

\section{Diagnóstico}

- Se realizaron las visitas pertinentes al caso, en las instituciones educativas dedicadas a la atención de los niños, niñas y jóvenes sordos.

- Reuniones con el personal directivo y docente, para ampliar las entrevistas y se recabó la información relacionada con el contexto de estudio

- Se llevó a cabo la elección de los grupos de estudio en el estado Aragua, a fin de recabar la información necesaria. El grupo de estudio estuvo conformado por 190 niños y jóvenes, cuyas edades estuvieron comprendidas entre 6 y 14 años respectivamente.

\section{Planificación}

- Luego de recabada la información y previo análisis de la información se elaboró el plan de acción, las actividades, los cronogramas y otros aspectos relacionados con la puesta en práctica de la propuesta de la I Olimpiada de lengua para niños y jóvenes Sordos en el estado Aragua. Esta actividad se organizó para dar respuesta a la misma preocupación relacionada con el uso de la lengua escrita en contextos significativos por parte de los niños sordos

- Con base en los postulados de la lingüística de la LSV, de la psicolingüística y la enseñanza del español escrito, se organizó el plan de acción y en ese marco, se ejecutaron reuniones de carácter informativo y formativo con los docentes, auxiliares, padres, representantes y comunidad educativa, así como también con los niños y jóvenes para facilitar su participación en el proyecto, lideradas por la investigadora y en compañía de los 8 (ocho) estudiantes universitarios.

- Se eligió el cuento El Sapo enamorado del autor Velthuijs (2009), debido a las características de la población y su relación con el texto.

- Se diseñaron los formatos a emplear durante la ejecución del plan de acción, así como guías de apoyo y orientación a los docentes para la puesta en práctica de las actividades.

\section{Ejecución}

- Se llevaron a cabo diversas visitas a las instituciones participantes en la investigación, con el fin de informar y orientar el trabajo a realizar durante la ejecución del plan de acción.

- Se presentó el cuento ante la comunidad, a partir de cuentacuentos desarrollados por personas sordas y oyentes en lengua de señas venezolana, así como por los mismos niños sordos.

- Se diseñó una presentación del cuento en diapositivas, así como también ejemplares del cuento.

- Se llevó a cabo la distribución del material y las ejercitaciones tipo diseñadas por la investigadora para la Olimpiada. 
- Se realizaron las inscripciones de las instituciones y los niños en las Olimpiadas.

- La investigadora diseñó el logo de la olimpiada, los tipos de pruebas y los formatos para su registro.

\section{Evaluación}

- La evaluación estuvo orientada hacia la participación de las comunidades educativas y hacia el logro de los objetivos propuestos en el plan de acción. Para realizar la evaluación se solicitó la opinión de los diferentes actores participantes durante la ejecución del plan de acción propuesto, así como la evaluación del proceso general por parte de la investigadora, de acuerdo con el logro de los objetivos.

\section{Resultados y discusión}

La concepción de la Olimpiada tuvo su base en las necesidades de quienes hacen vida en las instituciones de la Modalidad de Educación Especial del área de atención a personas sordas. Tales necesidades quedaron establecidas en el diagnóstico realizado en contraste con las investigaciones previas para su atención y las dificultades en su relación con el español escrito como segunda lengua. La Olimpiada, como investigación, fue concebida y organizada por la investigadora principal y la ejecución de las actividades se llevó a cabo en el marco de la Fase de Ejecución de Proyectos Educativos con la participación de ocho (8) estudiantes universitarios. Es importante destacar que la sistematización y organización de la investigación, así como el plan de acción, en rigor corresponde a la investigadora principal, pues la participación de los estudiantes en su oportunidad, fue de tipo operativa como parte de su formación.

El plan de acción cuyo objetivo general fue favorecer la producción y comprensión de textos escritos en español en niños y jóvenes Sordos, partió del respeto a la lengua de señas. El plan se desarrolló en un contexto de aprendizaje significativo y estimulante, mediante la organización de la I Olimpiada de lengua para niños y jóvenes sordos y tuvo como resultados los siguientes:

Para la valoración de la ejecución de la Olimpiada, se tomó el análisis y la reflexión como principio de la evaluación y un elemento transversal en forma de espiral durante para todo el proceso. Este proceso consistió en la revisión de todas las acciones, a lo largo de las fases de la investigación y de las etapas que comprendieron el plan de acción propuesto para dar vida a la Olimpiada. En tal sentido, la evaluación se desarrolló a partir del análisis de los siguientes insumos: 
registros de las actividades desarrolladas; notas de campo elaboradas por los investigadores; actividades desarrolladas y reportadas en los informes presentados por los estudiantes universitarios y las opiniones de los actores participantes (docentes, niños, personal directivo, padres y representantes).

Toda la información preveniente de las fuentes citadas se organizó y arrojó como resultado el resumen que decantó en la construcción a posteriori de categorías que surgen de la sistematización de toda la información como proceso de valoración y análisis. Estas categorías son la consecuencia natural que se muestra en las conclusiones de la investigación acción participante que se desarrolló en atención a los objetivos propuestos. En tal sentido, se describen a continuación los resultados de la valoración de la I Olimpiada:

En la Unidad Educativa:

-La investigación contó con la colaboración y disposición del personal directivo, docente, administrativo y obrero de las instituciones participantes.

-La participación de los niños surgió de manera inmediata al presentar el proyecto de la Olimpiada.

-Algunos docentes se mostraron escépticos ante la propuesta de la I Olimpiada, sin embargo, se incorporaron durante el desarrollo de las actividades.

- En un primer momento, los padres y representantes no se involucraron en las actividades, pero ante la insistencia de sus niños y jóvenes, se animaron a participar y asistir a la Olimpiada.

-Poca presencia de actividades significativas que faciliten el contacto del niño y joven sordo con el español escrito, desde su función social, tanto en la escuela como en el hogar.

-Preocupación en padres, representantes de los niños y jóvenes Sordos acerca del futuro académico, relacionado con la escasa producción y comprensión de textos escritos en español y sus oportunidades de estudio y trabajo.

- Las instituciones necesitan mejoras en la infraestructura, las cuales deben ser acondicionadas para ofrecer una educación de calidad a los niños y jóvenes Sordos. 
-Los docentes y personal de las unidades educativas participantes, trasladaron a los niños y jóvenes a la sede de la universidad, para garantizar su participación en la jornada final de la Olimpiada.

2.- Desarrollo de la I Olimpiada.

- Algunos de los estudiantes responsables de ejecutar las actividades de ejercitación incumplieron en algunas oportunidades sus responsabilidades.

- Problemas con el transporte desde el Municipio Libertador, para trasladar a los participantes, pero se solventó a través de servicios privados de transporte el día de la final de la Olimpiada.

- Tanto los docentes como los estudiantes necesitan profundizar en las investigaciones y estudios que dan cuenta del aprendizaje del español, así como actividades significativas que estimulen el uso del español escrito como segunda lengua en los niños sordos.

- En general, los estudiantes se comprometieron con la ejecución de proyecto de investigación y la concreción de la I Olimpiada.

-Levantar la información para la construcción del diagnóstico de la situación llevó más tiempo de lo previsto, aunado a las suspensiones de actividades escolares.

La valoración más importante guarda relación con el cumplimiento de los objetivos propuestos en el plan de acción y como mira más lejana la satisfacción de los propósitos de la investigación desarrollada, en tal sentido, mediante la ejecución de la planificación, se favoreció la producción y comprensión de textos escritos en español, partiendo del respeto a la lengua de señas, en un contexto de aprendizaje significativo y estimulante, mediado por las actividades relacionadas con I Olimpiada de lengua para niños y jóvenes Sordos en el estado Aragua.

En tal sentido, de acuerdo con la matrícula de cada institución, todos los niños y jóvenes sordos cuyas edades estaban comprendidas entre 6 y 14 años, pertenecientes a las unidades educativas del Municipio Girardot y Libertador participaron en las actividades relacionadas con la I Olimpiada, lo cual representa el logro del objetivo propuesto, en términos de cooperación. La distribución del material escrito, las bases y formas de participación, así como los criterios de 
evaluación en las instituciones participantes en la I Olimpiada de lengua para niños y jóvenes Sordos, se cumplió de acuerdo con lo estipulado.

El material teórico, bases y orientaciones a los docentes e instituciones fue redactado y presentado ante las autoridades de las instituciones, así como también ante los docentes, auxiliares, padres y representantes y miembros de la comunidad educativa que asistieron a las diversas actividades programadas. El cuento El Sapo Enamorado original, fue adquirido por la investigadora principal y se entregó a cada institución. Así mismo, se reprodujo todo el material de las ejercitaciones, así como los formatos de cada prueba, de acuerdo al grupo, los formatos para recabar la información durante las semifinales y final de la Olimpiada, por cuanto el objetivo se cumplió.

Por su parte, se ejecutaron las actividades pautadas de la I Olimpiada con el fin de favorecer la producción y comprensión de textos escritos en español, a partir del cuento el Sapo enamorado de Velthuijs (2009) se resumen a continuación:

1) Reunión del equipo de investigación con el jurado evaluador para la elección de los cuentos y material para las Olimpiadas.

2) Reproducción del material elegido para la I Olimpiada.

3) Reunión con Cuentacuentos en Lengua de Señas Venezolana.

4) Diseño, reproducción y distribución del material de trabajo para los diferentes grupos de participación.

5) Actividades especiales de Cuentacuentos en LSV en cada institución.

6) Ejercitación y práctica de los tipos de prueba para las olimpiadas, así como prácticas de dramatización y cuentacuentos desarrolladas por los niños y jóvenes Sordos.

7) Organización de los grupos por edades en cada institución educativa: Grupo A: de 6 a 8 años; "B" de 9 a 11 años y "C" de 12 - 14.

8) Organización del cronograma de actividades. Comunicación constante con los docentes y los estudiantes de la universidad.

9) Designación de comisiones de trabajo.

10) Observación y acompañamiento de las actividades en las unidades educativas.

11) Participación de las unidades operativas como colectivo, mediante la dramatización del cuento.

12) Ejecución de la I Olimpiada de lengua para niños y jóvenes sordos en el estado Aragua: una semifinal en cada instituto donde se organizó a los participantes para la final (5 por cada grupo, 15 por cada instituto) y la final en la Universidad Pedagógica Experimental Libertador. 
13) Elaboración y envío de las comunicaciones dirigidas a las instancias pertinentes, a fin de garantizar el espacio físico para realizar la Olimpiada, el sonido, la limpieza del espacio, el transporte, entre otros aspectos.

14) Participación de la moderadora.

15) Presentación de la Olimpiada en el marco de la II Jornada de reflexión de Práctica profesional.

16) Evaluación de la I Olimpiada.

Las actividades planificadas, se cumplieron mutatis mutandi, de acuerdo con el cronograma presentado ante el personal directivo y docente de las instituciones educativas participantes en la investigación. Para tal fin se reajustaron algunas fechas y se dio cumplimiento a las actividades previstas, completando el logro de los objetivos propuestos. La figura 1, resume de manera esquemática el proceso de ejecución de la I Olimpiada de lengua para niños y jóvenes Sordos, en el cual se concretó la construcción de enunciados en forma escrita y en lengua de señas acerca de los textos trabajados para facilitar la participación en la olimpiada, así como la dramatización y puesta en escena de los mismos. 


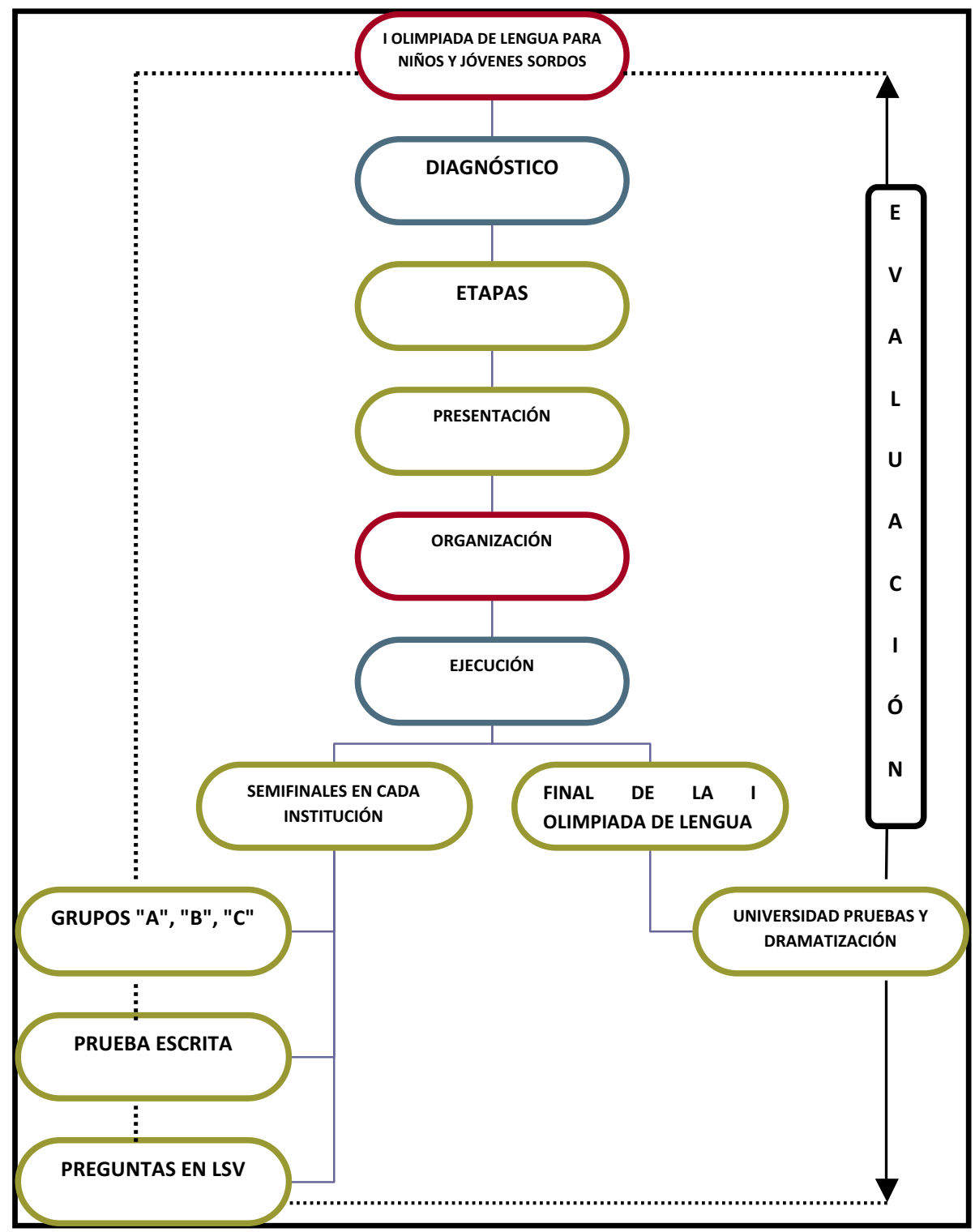

Figura 1. Ejecución de la I Olimpiada para niños y jóvenes Sordos en el estado Aragua

Luego de clasificar la información y de acuerdo con las categorías que surgieron del análisis de la información proveniente de las diferentes fuentes, se puede afirmar que la experiencia de los niños y jóvenes Sordos en la Olimpiada, resultó significativa y mejoró su interés en la lengua escrita. Más allá de la investigación la mejor evidencia son las expresiones de alegría de los niños 
y la satisfacción de los padres, representantes, familiares, docentes y personal directivo de los planteles que asistieron a las actividades.

La actitud inicial de rechazo y desgano ante la lectura y escritura en el aula, se superó día a día con las actividades lúdicas planificadas para la realización de la Olimpiada, los niños quienes en un primer momento se mostraron temerosos y escépticos ante el texto escrito, trabajaron poco a poco el vocabulario, emplearon su lengua para comunicarse, construyeron y reconstruyeron el texto El sapo enamorado (2009), dramatizaron sus acciones y contagiaron de entusiasmo a sus docentes y padres alrededor de esa narración, su reflexión y sus enseñanzas. La Olimpiada como punto de inicio para la comunidad participante, se constituyó como un camino posible hacia la construcción de significados a partir de textos escritos, en los niños y jóvenes Sordos, como proyecto educativo.

\section{Conclusiones}

La historia de la Educación Especial en Venezuela y el devenir de los distintos modelos pedagógicos que han dado cuenta de la atención de las personas con discapacidad a lo largo de más de 50 años, ubica a las personas Sordas en una dimensión de respeto a sus necesidades comunicativas y condición lingüística como comunidad, a partir de la visión socioantropológica contenida en el Modelo de Atención Educativa Integral del Deficiente Auditivo Venezolano. Este modelo, puesto en marcha por el Ministerio de Educación en 1998-99, desde el punto de vista legal, se encuentra vigente en el país. No obstante, la aplicación del modelo, no satisface completamente las necesidades e intereses de los estudiantes Sordos en términos de su relación con los textos escritos en español, debido a los factores sociolingüísticos, educativos y políticos que han obstaculizado el fortalecimiento de una educación verdaderamente bilingüe-bicultural. Una educación verdaderamente bilingüe, debe garantizar el acceso a la información y a la formación de los niños y jóvenes Sordos, en su lengua natural, la lengua de señas; la atención familiar y social que le garantice la comunicación fluida en ese código en los contextos cotidianos de su vida diaria, además de recibir una formación que les garantice la producción y comprensión de textos escritos en español en contextos reales y significativos de aprendizaje, con base en la función social de la lengua escrita.

Tal y como se argumentó suficientemente en el diagnóstico y a lo largo de las referencias que sirven de antecedentes y sustento teórico a esta investigación, la relación de la población Sorda en 
el país con la lengua nacional en forma escrita, resulta escasa o insuficiente para el logro de metas personales, académicas y sociales de este grupo lingüístico minoritario. De allí que el estudio tuvo como propósito general la organización de actividades que estimulen el uso del código escrito como segunda lengua en los niños y jóvenes sordos.

Por ello, se diseñó, planificó y evaluó un plan de acción que apuntó a la ejecución de la Primera Olimpiada de la Lengua para niños y jóvenes Sordos con el objeto de facilitar su relación con los textos escritos en español, que partió del respeto a la lengua de señas, la participación y formación activa de los docentes, padres, representantes y comunidad, en un contexto de aprendizaje significativo y estimulante. En ese contexto se potenció la relevancia de la lengua de señas como código de comunicación y mediación pedagógica, familiar y social con los niños y jóvenes sordos.

La Olimpiada se llevó a cabo con la participación de la población estudiantil de las unidades operativas correspondientes al área de Sordos, ubicadas en el estado Aragua, Venezuela; para ello se contó con el personal docente oyente y Sordo, padres, representantes y comunidad en general, con quienes a través de la formación e información se organizaron las jornadas de trabajo para acercar los textos escritos en español a la comunidad escolar sorda, durante el año escolar 20122013. Este proceso decantó como producto del análisis, en un cuerpo conclusiones, que se lograron mediante la contrastación de los objetivos, las categorías y los referentes teóricos empleados en el estudio, las cuales se presentan a continuación:

La indagación de los contextos de estudio, conformados por las dos unidades educativas dedicadas a la atención de niños y jóvenes sordos, participantes, reportó la precaria realidad de sus infraestructuras y condiciones generales, que a pesar de ello cuentan con la mística y el compromiso para garantizar la calidad educativa de la población que atienden. Estas comunidades hacen su mejor esfuerzo, a pesar de las necesidades, por facilitar el camino hacia una educación con equidad de oportunidades que tienda a mejorar su comunicación en la lengua de señas venezolana, lengua en la que se comunican con fluidez sus estudiantes.

En el personal que labora en las unidades educativas participantes en el estudio, existe la genuina preocupación por la escasa relación entre los niños y jóvenes Sordos y el código escrito, pues a pesar de sus esfuerzos al diseñar estrategias y ejecutar actividades, no se cumplen a cabalidad los objetivos propuestos pues resulta evidente que ellos continúan sin apropiarse del código escrito, 
tal y como lo plantean, Luque, (1994) y (2004); Morales y Vallés (2002), Zambrano (2008). Esta información resulta coherente con el diagnóstico que se elaboró en ambas instituciones, decantando en la imperiosa necesidad de planificar actividades que promuevan la participación de la población Sorda donde se favorezca su relación con el código escrito como segunda lengua. Por ello, la planificación y ejecución de la I Olimpiada de lengua para niños y jóvenes Sordos fomentó la participación las unidades educativas Maracay y Libertador, pues se propuso favorecer la producción y comprensión de textos escritos en español y para ello, partió del respeto a la lengua de señas, en un contexto de aprendizaje significativo y estimulante para ellos. En el marco de las actividades, los participantes consolidaron la caracterización de los cuentos y la estructura que presentan, lo cual sirvió de base para la comprensión y posterior construcción de este tipo de texto narrativo (Braslasky, 2005). Se partió para ello, de la reconstrucción del cuento, su vocabulario, se dramatizó el cuento siguiendo sus acciones y se contó en todo momento con el respeto a la lengua de señas y a sus características lingüísticas, lo cual garantizó su comprensión.

Las actividades planificadas que decantaron en la ejecución de la Olimpiada, contribuyeron a acercar las relaciones familia, escuela y comunidad, así como estrechar los lazos entre los niños y jóvenes sordos, sus docentes, padres y representantes y estudiantes universitarios para lograr un proyecto común. Las actividades desarrolladas, no sólo contribuyeron a la formación de nuevos docentes en el área de sordera, la incorporación de los padres y representantes en las actividades, , dado el entusiasmo demostrado por sus hijos ante un proyecto de lectura y escritura, que privilegió la función social de la lengua escrita dentro y fuera del aula (Smith, 1995).

La Olimpiada favoreció la incorporación de los padres y representantes en las actividades propuestas, lo cual se tradujo en un mejor rendimiento de los niños y jóvenes en las otras áreas del trabajo escolar, a partir de los compromisos establecidos entre ellos. Además, permitió la reflexión de los docentes, padres y representantes en cuanto a la importancia de la lengua de señas venezolana, el español como segunda lengua y el tipo de texto narrativo. Esta reflexión se produjo, mediante la toma de conciencia acerca de las diferencias lingüísticas entre la lengua de señas y el español escrito, y de la importancia de la lengua de señas venezolana en el desarrollo integral de los Sordos y el acompañamiento y apoyo que requieren en el hogar para el logro de sus metas. 
Esta reflexión metalingüística (Bruzual, 2002) permitió a los niños y jóvenes participantes comprender la diferencia entre decodificar y construir significados a partir de un texto escrito, y entre codificar y construir textos significativos en español. Así como también la importancia del proceso de planificar, textualizar y revisar la producción escrita, de acuerdo con los pasos para la producción escrituraria propuesta por Cassany $(1989 ; 1998 ; 1999)$, en tanto que la toma de conciencia acerca de las reglas ortográficas se abordó en las actividades diarias, a medida que iban surgiendo.

Los niños y jóvenes Sordos, por su parte, participaron con entusiasmo en las actividades, se logró despertar su interés en el proceso de construcción de la lengua escrita, que se llevaron a cabo con apoyo de los docentes de aulas y de algunos padres y representantes desde el hogar, en atención a los principios planteados por Schepler (1997): Al leer el cuento, los lectores sordos amplían la información, no solamente traducen lo que aparece en el cuento, sino que también explican. Los niños y jóvenes recrearon con libertad el cuento, disfrutaron su lectura y dramatización, lograron comprender y no solo memorizar y repetir.

Las actividades planificadas y ejecutadas en la Olimpiada, mejoraron la relación de los niños y jóvenes Sordos con el código escrito como segunda lengua en cuanto a la producción y comprensión de textos; promovieron la participación de los padres en los procesos de lectura y escritura de sus hijos; mejoraron el rendimiento académico de los participantes y las relaciones interpersonales así como el desempeño de los docentes de aula y por ende el fortalecimiento de la educación para la paz y respeto a la diversidad.

Como consecuencia, este trabajo de investigación favoreció la formación de los estudiantes, la comunidad educativa y la comunidad científica en el campo de la investigación de lingüística aplicada. De allí, que la mira más lejana de la investigación consistió en promover y concretar la ejecución de la Olimpiada de Lengua para niños Sordos, como piloto innovador que pueda desarrollarse en otros ámbitos a nivel nacional e internacional.

El valor de este trabajo radica en la nueva relación que establecieron los niños y jóvenes sordos con el código escrito como segunda lengua. Esta relación se basó en la producción y comprensión de textos desde la función social que tiene la lengua nacional en su desarrollo integral. La incorporación de los padres, representantes y familiares en los procesos de lectura y escritura de 
sus hijos; el rendimiento académico de los estudiantes universitarios de la especialidad de Educación para sordos en el Estado Aragua y su acercamiento a la realidad escolar; la reflexión y acción del docente que día a día debe favorecer la producción y comprensión de textos escritos en español; la importancia de la lengua de señas y el respeto a sus características lingüísticas; la puesta en práctica de actividades innovadoras que propicien un contexto de aprendizaje significativo y estimulante, dedicado a satisfacer las necesidades y potencialidades de expresión para niños y jóvenes Sordos.

Finalmente, resulta evidente que las actividades propuestas en este estudio promueven la participación activa de las comunidades educativas del área, en actividades innovadoras que promueven la unión y fortalecen la relación entre la familia, escuela y comunidad con base en el respeto, el amor y la promoción de la paz.

\section{Referencias}

Braslawsky, B. (2005) Enseñar a entender lo que se lee. La alfabetización en la familia y en la escuela. Argentina: Fondo de Cultura Económica de Argentina.

Bruzual, R. (2002). Propuesta comunicativa para la enseñanza de la lengua materna. Vicerrectorado Académico Universidad del Zulia (LUZ). Venezuela: Data. Maracaibo.

Camps, A. (2002). La enseñanza y el aprendizaje de la lengua escrita. En Lomas, Carlos (Comp.), El aprendizaje de la comunicación en las aulas (112-144). Barcelona. Paidos.

Cassany, D. (1989). Describir el Escribir. Cómo se aprende a escribir. España: Paidos.. (1998). La cocina de la escritura. Sexta edición. España: Anagrama. (1999). Construir la escritura. Barcelona: Paidos.

Cisterna , F.(2005). Categorización y triangulación como procesos de validación del conocimiento en investigación cualitativa. Theoria, 14(1), undefined-undefined. [fecha de Consulta 23 de Octubre de 2019]. ISSN: 0717-196X. Disponible en: https://www.redalyc.org/articulo.oa?id=299/29900107

Hurtado, I. y Toro, J. (1997). Paradigmas y métodos de investigación en tiempos de cambio. Venezuela: Episteme Consultores y Asociados.

Ley para Personas con Discapacidad (2007). Venezuela. 
Luque, B. (1994). Estrategias para facilitar la lectura en los niños sordos. Trabajo de grado de maestría no publicado. Venezuela: PEL. IPC.

(2004). Creación de un Laboratorio de Lengua de Señas Venezolanas. Trabajo de Ascenso no publicado. Caracas: UPEL. IPC

Martínez, M. (1999). La investigación Cualitativa etnográfica en Educación. Manual Teórico Práctico. México: Trillas

Martínez, M. (2011). Ciencia y arte en la metodología cualitativa. México: Trillas

Ministerio de Educación. (1998) Conceptualización y Política de la Atención Educativa Integral del deficiente Auditivo. Caracas: EF

Morales, A y Vallés, B. (2002). Análisis de los fenómenos sintácticos en la escritura de adolescentes sordos venezolanos. Caracas: FE Experimental Libertador.

Sánchez, C. (2014) Los sordos: una comunidad secuestrada. Disponible en: http://www.culturasorda.eu. Fecha de consulta: 23/09/2019.

Schleper, D. (1997). Estrategias de Prelectura. Estados Unidos: Gallaudet University. Washinton.

Smith, F. (1995). Comprensión Lectora. Análisis psicolingüistico de la lectura y su aprendizaje.. México: Trillas

Teppa, S. (2006). Investigación Acción participativa en la praxis pedagógica diaria. Barquisimeto: UPEL-IPB

Velthuijs, M. (2009). El sapo enamorado. Caracas: Ekaré.

Zambrano, L (2008) Tipología de textos escritos en español por sordos. Revista de Educación: Laurus. Año 14, No 26. Caracas, Venezuela.

\section{Para citar este artículo:}

L. Zambrano y L. Díaz (2019). Olimpiada de lengua para sordos una experiencia lingüística y educativa. Revista Educa UMCH, 14(1). https://doi.org/10.15756/educaumch.v7i13.97 
Educa UMCH. Revista sobre Educación y Sociedad, 2019, 14(1), 5-22.

https://doi.org/10.35756/educaumch.v0i14.116

Contacto: revistaeduca@umch.edu.pe_ISSN : 2617-0337 revistas.umch.edu.pe/ 